\title{
La autocita en artículos de investigación publicados en Scielo Chile: variación según el área de la ciencia y la experticia del autor
}

\author{
Paulina Meza* \\ Gustavo Ortega**
}

Artículo recibido:

8 de enero de 2019

Artículo aceptado:

25 de marzo de 2019

Artículo de investigación

\section{Resumen}

El objetivo de esta investigación fue describir el uso de la autocita en artículos de investigación publicados en Scielo Chile, de diversas áreas de la ciencia, escritos por autores con distintos niveles de experticia en el ámbito de las publicaciones científicas. A partir de una investigación inductivo-deductiva, analizamos un corpus de 182 artículos de cinco áreas de la ciencia, escritos entre 2010 y 2014 por autores noveles y consolidados. Entre los principales resultados destacamos que Humanidades lidera la frecuencia de uso de autocitación, mientras que Ciencias Médicas es la disciplina en la que la autocita es menos común. Esto

\footnotetext{
Instituto de Investigación Multidisciplinar en Ciencia y Tecnología, Universidad de la Serena, Chile pmeza@userena.cl

** Instituto de Literatura y Ciencias del Lenguaje, Pontificia Universidad Católica de Valparaíso, Chile gustavortega.s@gmail.com

INVESTIGACIÓN BIBLIOTECOLÓGICA, vol. 33, núm. 81, octubre/diciembre, 2019, México, ISSN: 2448-8321 pp. 41-56
} 
podría atribuirse a que los autores de Ciencias $\mathrm{Hu}$ manas requieren mayor difusión de sus trabajos, por lo que la autocita sería una forma simple y rápida para aumentar los índices de citación y la visibilidad de sus investigaciones. En cuanto al nivel de experticia, los autores más dados a autocitarse son los consolidados. En conclusión, creemos que la autocitación constituye una estrategia de posicionamiento del autor, ya sea para mostrar sus trabajos previos, evidenciar su línea investigativa, aumentar sus índices, lograr visibilidad o simple vanidad.

Palabras clave: Artículo de Investigación; Scielo Chile; Autocita; Variación Disciplinar; Variación de Experticia

Self-citation in research articles published in Scielo Chile: variation according to the field of science and the expertise of the author

Paulina Meza and Gustavo Ortega

\section{Abstract}

The objective of this analysis was to describe the use of self-citation in research articles published in Scielo Chile, written by authors from different scientific areas and with different levels of expertise in the field of scientific publications. Based on an inductivedeductive approach, we analyzed 182 articles from five areas of science, written between 2010 and 2014 by novice and expert authors. The main results show that the field of Humanities leads the frequency of self-citations, in opposition to the field of Medical Sciences, where self-citations are less common. These results could be attributed to the fact that the authors of Human Sciences require greater dissemination of their work, being the self-citation a simple and quick way to increase the citation indexes and the visibility of their research. Regarding the level of expertise, the authors presenting more self-citations are the expert ones. In conclusion, we believe that self-citation is a positioning strategy for authors in order to show previous works, demonstrate a research line, increase his/ her impact values or achieve visibility, or simple vanity.

Keywords: Research Article; Scielo Chile; Selfcitation; Discipline Variation; Expertise Variation 


\section{INTRODUCCIÓN}

$\mathrm{L}$ a citación constituye una práctica común y necesaria en la publicación de cualquier artículo de investigación (en adelante AI), puesto que otorga objetividad al discurso científico (Sabaj y Páez, 2010) y permite al autor contextualizar y vincular temáticamente su investigación. Si bien en la escritura académica y científica es posible identificar diversos tipos de citas, son las autocitas las más controversiales desde que la reputación profesional de los investigadores ha sido mediada por el número de publicaciones y por el impacto de las revistas en las que publicasen (Gul, Shah y Shafiq, 2017).

Para algunos, la autocita permite demostrar la vinculación temática de las investigaciones y reafirmar la autoridad investigativa del autor, por lo tanto, sería una práctica necesaria en el discurso científico (Campanario y Candelario, 2010; Gálvez, 2017; Reiter, Mimouni y Mimouni, 2015). En contraposición, hay quienes plantean que la finalidad de las autocitas es la autopromoción y el aumento del factor de impacto de las revistas (Deschacht y Maes, 2017; Seeber et al., 2017). La autocita sería, por tanto, un vicio innecesario o práctica cuestionable que surge como respuesta estratégica a las métricas de publicación (Anderson, Seashore y Earle, 1994) y para alcanzar el reconocimiento de los pares y de la comunidad.

En cualquier caso, se desconoce, primero, cuáles son los tipos y funciones concretas de la autocita en los AI; segundo, si la disciplina y el grado de experticia del autor inciden en su utilización. Hasta la fecha, las investigaciones se han centrado en estudiar las autocitas desde parámetros netamente bibliométricos (George-Nascimento, 2010; Salvador-Oliván, Marco-Cuenca y Arquero-Avilés, 2018) sin considerar la variación disciplinar ni la experticia del autor.

En este marco, nos preguntamos: ¿existe diferencia en el uso de la autocita en AI según el área de la ciencia y el grado de experticia del autor? Para dar respuesta a esta interrogante, planteamos como objetivos: a) describir el uso de la autocita en AI de diversas áreas de la ciencia y escritos por autores con distintos grados de experticia, y b) determinar la variación de la autocita en el género AI según dichas variables.

\section{Antecedentes: estudios sobre autocitas en AI}

Para Ganga, Paredes y Pedraja-Rejas (2015), uno de los medios más utilizados, rigurosos y efectivos para transmitir el conocimiento disciplinar es el AI, por lo que reflexionan sobre los principales errores que cometen los investigadores al publicar. Algunos de ellos son problemas de contexto (falta de rigor en el cálculo del FI, índice de inmediatez y país de origen de las 
publicaciones) e inconvenientes ligados a las revistas (demora en la publicación, incompetencias en la revisión de artículos, abuso de autocitas, etc.). De este último grupo, dichos autores afirman que el uso de la autocita con fines de continuidad lógica-investigativa no sería una práctica cuestionable. El problema surge cuando se hace uso excesivo de ella sin un propósito académico o científico claro, o con la única intención de subir el FI. Sin embargo, otros autores, mediante estudio de corpus, afirman que no existe relación entre el número de autocitas y el aumento del FI, por lo que la autocita no sería una práctica científica cuestionable (Campanario y Candelario, 2010; Reiter, Mimouni y Mimouni, 2015).

Gálvez (2017), por su parte, estimó una métrica de captura semántica entre los AI que le permitió corroborar que las autocitas tienden a conectar trabajos que comparten contenido semántico. Es decir, constituyen una herramienta que posibilita guiar al lector en la búsqueda y relación de temáticas. Según este estudio, entonces, el uso de autocitas estaría justificado, al menos temáticamente.

Desde un plano sociológico, Deschacht y Maes (2017) investigan la variación de las autocitas según el campo cultural del autor. A partir del análisis de 1346 artículos de Ciencias y Negocios, concluyeron que los autores de culturas individualistas son más propensos a autocitarse que los de culturas colectivas. Asimismo, los hombres se autocitan más que las mujeres, aunque este efecto de género es menor que el cultural.

Henao, Calvo y Gallego (2017) realizan un estudio longitudinal de citas y autocitas en revistas colombianas entre 2007 y 2015. A diferencia de nuestra investigación, en este trabajo sólo se consideró ingeniería y la búsqueda se realizó de forma automatizada únicamente en la sección Bibliografía. Tras la investigación concluyen que, en la revista que es el foco de la investigación, el número de citas recibidas presenta una tendencia a la baja, lo que se debería a la disminución de las autocitas.

\section{Metodología}

Los objetivos generales de esta investigación son los siguientes: a) describir el uso de los mecanismos de autocitación en AI de diversas áreas de la ciencia y escritos por autores con distintos grados de experticia, y b) determinar la variación del uso de dichos mecanismos en el género AI, según dichas variables. Las áreas incluidas son Ciencias Médicas y de Salud, Ciencias Naturales, Ciencias Sociales, Humanidades e Ingeniería y Tecnología, clasificación tomada de la OCDE (2007), cuya elección obedece a los argumentos esgrimidos 
en Meza (2017a). Los grados de experticia, por su parte, son autores noveles, esto es, que publican por primera vez en una revista indexada, y autores consolidados, aquellos que cuentan con más de una publicación en el mismo tipo de revista.

La metodología es similar a la utilizada en la investigación mayor en la que se enmarca este estudio (Meza, 2017b; Meza, Sabaj y Matsuda, 2017; Meza, 2018). Concretamente, se trata de un trabajo mixto, de alcance descriptivo y diseño no experimental (Hernández, Fernández y Baptista, 2006; Creswell, 2007). Para la obtención de datos, seguimos un enfoque ascendente-descendente (bottom up-top down) pues, al realizar el análisis, asumimos una postura abierta e ingenua frente a los textos analizados. En otros términos, no aplicamos modelos preconcebidos para identificar los mecanismos de autocitación en el corpus, sino que han sido identificados a partir de la lectura y análisis de los textos del corpus. Al mismo tiempo, los investigadores poseen conocimientos previos que no pueden dejar de lado, por lo que no es un estudio puramente inductivo (Tognini-Bonelli, 2001).

El corpus analizado, denominado CORAI ACEX (Corpus Representativo de Ârtículos de Investigación por Áreas de la Ciencia y Grados de Experticia), se conformó primeramente atendiendo dos criterios de inclusión:

a) AI publicados en español por un solo autor entre 2010 y 2014. Optamos por AI de un solo autor debido a que consideramos la autocita como un acto retórico individual. Por tanto, al incluir AI publicados por un autor único, evitamos posibles confusiones con ciertas marcas lingüísticas de autocitación y, además, nos permite hacer comparaciones sin que influya la variable cantidad de autores por artículo.

b) AI disponibles en Scielo Chile, lo que no implica que todos los artículos son de investigadores chilenos, sino que son autores de distintas nacionalidades que han publicado en revistas disponibles en Scielo Chile.

Los AI que cumplieron con ambos criterios fueron 2 938. A partir de este número, calculamos la muestra mediante un muestreo estratificado con afijación proporcional, el cual asegura que todos los estratos estén correctamente representados (Martínez y Martínez, 2008). El nivel de precisión calculado fue de $7 \%$. Además, para seleccionar la muestra de cada estrato se utilizó un generador de números aleatorios sin repetición.

A partir de la aplicación de dichos procedimientos, la muestra quedó conformada como se indica en la Tabla 1. 


\begin{tabular}{|l|l|c|c|}
\hline \multicolumn{1}{|c|}{ Área de la ciencia } & Grado de experticia & Total & Total \\
\hline \multirow{2}{*}{ Ciencias Médicas y de Salud } & Novel & 10 & \multirow{2}{*}{12} \\
\cline { 2 - 3 } & Consolidado & 2 & \\
\hline \multirow{2}{*}{ Ciencias Naturales } & Novel & 4 & \multirow{2}{*}{5} \\
\cline { 2 - 3 } & Consolidado & 1 & \multirow{2}{*}{87} \\
\hline \multirow{2}{*}{ Ciencias Sociales } & Novel & 67 & \multirow{2}{*}{76} \\
\cline { 2 - 3 } & Consolidado & 20 & \multirow{2}{*}{2} \\
\hline \multirow{2}{*}{ Humanidades } & Novel & 59 & \\
\cline { 2 - 3 } & Consolidado & 17 & \\
\hline \multirow{2}{*}{ Ingeniería y Tecnología } & Novel & \multicolumn{2}{|c}{182} \\
\cline { 2 - 3 } & Consolidado & \multicolumn{2}{|c}{} \\
\hline \multirow{2}{*}{ Total } & & \multicolumn{2}{|c}{} \\
\hline
\end{tabular}

Tabla 1. Conformación de la muestra

Se observa que no contamos en el corpus con textos de autores consolidados de Ingeniería puesto que, luego de aplicar los criterios de inclusión mencionados, dicho estrato no quedó representado. Una vez determinada la muestra, considerando el tamaño de cada estrato, dividimos CORAI ACEX en un corpus de prueba ( $33 \%$ de los textos) y uno de investigación (67\% restante). Con el corpus de prueba levantamos, testeamos y ajustamos una versión inicial del análisis. A su vez, en el corpus de investigación, tomamos como punto de partida las categorías identificadas en el corpus de prueba, las que fueron validadas mediante un juicio de pares expertos, aunque sin cerrarnos a la posibilidad de que pudiesen aparecer nuevas categorías emergentes. Las categorías de análisis o, más concretamente, los mecanismos de autocitación identificados los presentamos en el siguiente apartado, pues, debido al método de obtención de datos utilizado, constituyen uno de nuestros resultados. El etiquetaje de las categorías fue realizado con el software Atlas.ti (versión 7.5.12).

Además de la separación entre un corpus de prueba y uno de investigación, utilizamos los siguientes mecanismos de validación en cada etapa del análisis de ambos subcorpus:

a) Triangulación. Una vez obtenidas las categorías de análisis (tanto las iniciales del corpus de prueba como las emergentes del corpus de investigación), se entrenó a tres asistentes, quienes analizaron algunos artículos del corpus con el propósito de corroborar que el etiquetaje de las categorías no fuese subjetivo. Todos los casos que provocaron algún tipo de incertidumbre fueron revisados en conjunto hasta 
despejar cada duda. De este modo, pudimos cerciorarnos de la existencia de acuerdo en el análisis realizado.

b) Revisión de las categorías y método de análisis. Se consultó a dos investigadores (uno chileno y uno extranjero), ambos doctores en lingüística, hablantes nativos de español, expertos en escritura académica y científica.

c) Juicio de evaluadores expertos. Los jueces seleccionados, tanto para la validación de $33 \%$ como de 100\% del corpus, son doctores en lingüística, hablantes nativos de español, especialistas en escritura académica o científica, chilenos y extranjeros.

El análisis del corpus de prueba y del de investigación superaron el valor de referencia mínimo para la validación mediante el porcentaje de acuerdo, que es de 70 \% (Blessing y Chakrabarti, 2009). En cuanto a la aplicación del coeficiente Kappa, y siguiendo la interpretación de los valores propuestos por Landis y Koch (1977), obtuvimos para el corpus de prueba un acuerdo considerable (0.67) y para el corpus de investigación un acuerdo casi perfecto (0.91). Estos cálculos fueron realizados con la herramienta ReCal3: Reliabilityfor 3+ Coders (Freelon, 2010).

\section{ANÁLISIS Y DISCUSIÓN DE RESULTADOS}

Como mencionamos anteriormente, los mecanismos de autocitación identificados en esta investigación constituyen nuestro primer resultado. En concreto, identificamos en CORAI ACEX dos tipos de mención de los propios trabajos (o autocitas) que constituyen, por cierto, nuestras dos categorías de análisis: la autoreferencia y la autorecomendación. La primera la entendemos como la referencia que hace el autor a trabajos propios en su AI. Nos alejamos, por tanto, de la noción de autoreferencia (self-reference) que poseen otros autores como Hyland (2003), quien la entiende como la alusión al autor a través de marcas lingüísticas específicas de primera persona singular. Un ejemplo de nuestra concepción de autoreferencia es el siguiente:

Por otra parte, las diferencias de los procedimientos de administración aplicado [sic] entre los varios tipos y magnitudes de las obras monitoreadas, a nivel proyecto, tienen un efecto menor en el nivel de la observación de la actividad, mientras que los posibles impactos de otros factores que intervienen, tales como: tamaño y composición de la cuadrilla, habilidades de la mano de obra, motivación y supervisión de la calidad pueden ser moderados, recopilando un volumen mayor de datos de productividad laboral (Jarkas, 2005) [IYT_F_2. Autor AI: Abdulaziz M. Jarkas]. 
Por otro lado, a través de la autorecomendación, el autor indica directamente al lector que revise sus escritos, los que no siempre tienen relación directa con el tema tratado en el artículo donde presenta dicha recomendación. En este sentido, no se cumpliría en nuestro corpus el hallazgo de Gálvez (2017), quien señala que las autocitas no son irrelevantes, sino que guardan estrecha vinculación con el contenido trabajado. Un ejemplo de autorecomendación es: "Para mi punto de vista acerca de los derechos que merecen ser tratados como derechos democráticos, véase Munck (2007, 2009b: 343-45, 2009c)" [CS_C_788b. El autor del AI es Gerardo L. Munck].

Como se puede apreciar en los ejemplos, en el primer caso se hace referencia a un trabajo propio de la misma temática con el propósito de sustentar una afirmación incluida en el artículo. En cambio, en el segundo caso el autor remite al lector a otra de sus publicaciones, que no necesariamente tiene una vinculación con el tema abordado, a fin de que revise su punto de vista acerca de un tema determinado. Ambos tipos corresponderían a una categorización más específica de lo que Hyland (2003) denomina autocita directa, en la que el investigador referencia trabajos de su autoría con el objetivo de enfatizar la contribución personal de su investigación.

En adelante, para obtener los resultados numéricos, calculamos el promedio de categorías de autocita según la cantidad de AI que componen el corpus o cada estrato del corpus, según sea el caso. Así, en total en CORAI ACEX encontramos 400 casos de autocita (autoreferencia más autorecomendación), lo que significa que hay una presencia promedio de 2.2 autocitas por cada artículo. En otras palabras, en promedio, los autores se autocitan más de dos veces en cada texto. En un análisis más específico, advertimos que hay un promedio de 1.81 autoreferencias y 0.38 autorecomendaciones por cada texto. Estos valores representan la cantidad de veces que se menciona a sí mismo cada autor en su AI, independientemente de si se trata de una misma publicación que se referencia varias veces a lo largo del texto o si corresponde a publicaciones distintas del mismo autor.

En la Figura 1 mostramos cómo se presentan las categorías mencionadas en el corpus analizado, según la variable área de la ciencia.

Un primer aspecto interesante es el hecho de que, aunque con distinta frecuencia, la autoreferencia es transversal a todas las áreas estudiadas; no así la autorecomendación, que es exclusiva de algunas de ellas, concretamente, de Ciencias Sociales ( 0.46 casos por AI) y Humanidades ( 0.39 casos por AI). Consideramos que el uso de la autorecomendación, desde un punto de vista social, revela un ethos especial del investigador, pues va un paso más allá recomendando explícitamente sus propias publicaciones. En este sentido, 
creemos que la autorecomendación podría ser catalogada como una especie de atajo para aumentar las métricas del autor y el impacto de la publicación, pues en el corpus analizado no es un tipo de autocita que tenga como función contextualizar la investigación propia en tiempo y lugar (Copez-Lonzoy y Paz-Jesús, 2018) o sustentar la continuidad de un ámbito investigativo. Por el contrario, en la mayoría de los casos, se incita a la audiencia a leer un trabajo propio sobre un tema inconexo o vinculado sólo indirectamente con la investigación presentada. A modo de ejemplo, comentamos el caso de un AI que aborda el tema de la relación funcional entre el dolo y la consciencia de la antijuricidad en el derecho penal chileno donde, aprovechando una brevísima explicación relacionada con la teoría de los actos de habla, se incorpora la siguiente autorecomendación: "Para una revisión panorámica de las categorías fundamentales de la teoría analítica de los actos de habla, véase Mañalich, J.P., “¿Reglas del entendimiento? Acerca de la ‘juridificación’ de la comunicación en la pragmática universal de Jürgen Habermas”, en Estudios Públicos 119, 2010, pp. 127 ss., 135 ss., con ulteriores referencias” [CS_C_1152].

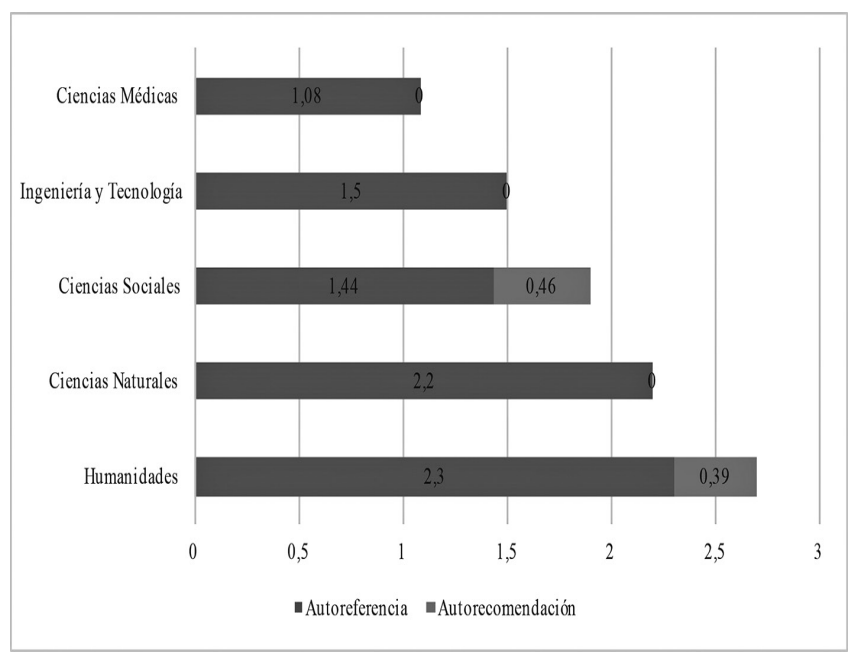

Figura 1. Promedio de autocitas según el área de la ciencia

Cabe agregar que el autor del AI del ejemplo incorporó 16 autorecomendaciones en un trabajo de 29 páginas, esto es, más de dos exhortaciones a leer trabajos propios por cada página. Este abultado número podría ser un indicador de que la autocita y, en particular la autorecomendación, sería una especie de respuesta o estrategia para enfrentar en mejores condiciones carreras académicas basadas en métricas. Lo anterior respondería a la necesidad 
de los investigadores por ser aceptados en la comunidad discursiva a la que pertenecen y, así, tener una mayor aprobación entre sus pares (Gul, Shah y Shafiq, 2017; Ganga, Paredes y Pedraja-Rejas, 2015).

Como muestra la Figura 1, esta práctica de autorecomendarse es exclusiva de áreas que se han agrupado bajo el rótulo de ciencias humanas o soft sciences (Humanidades y Ciencias Sociales), pero no aparece en las llamadas ciencias puras o hard sciences (Ciencias Médicas, Ciencias Naturales e Ingeniería y Tecnología). Por tanto, la presencia de la autorecomendación es una categoría que permite agrupar y, al mismo tiempo, diferenciar tipos de áreas de la ciencia. Creemos que su uso es propio de las ciencias humanas debido a que en ellas, tradicionalmente, la necesidad de investigación ha sido más difícilmente demostrable, por lo que los autores recurren a distintas estrategias para posicionar sus trabajos, entre ellas, el uso de autocitas para lograr aumentar su índice $b$ y, así, lograr renombre en su comunidad. Esta idea es compartida por diversos autores que afirman que dicho índice es susceptible de ser manipulado y una forma común de lograrlo es, precisamente, mediante el abuso de autocitaciones (Deschacht y Maes, 2017; Carlisle et al., 2017; Seeber et al., 2017; Copez- Lonzoy y Paz-Jesús, 2018).

Si consideramos la autocita como la suma de la autoreferencia y la autorecomendación, Humanidades lidera en frecuencia de uso de dicho mecanismo (2.69 casos en promedio por AI); mientras que, en el otro extremo, Ciencias Médicas es la disciplina en la que la autocita es menos común (1.08 casos en promedio por AI). Este resultado podría explicarse por el hecho de que son los autores de ciencias humanas quienes requieren mayor visibilidad de sus trabajos, por lo que recurren al uso de autocitas para hacerlo, pues, según Fowler y Aksnes (2007), cada autocita generaría 3.65 citas adicionales. Así, el uso de autocitas sería la forma más simple y rápida para aumentar los índices de citación y la visibilidad de los propios trabajos, por lo que su utilización podría considerarse una estrategia de posicionamiento.

La tendencia que hemos venido comentando persiste al revisar la presencia de la autoreferencia en el corpus, pues es Humanidades el área en que más frecuentemente aparece ( 2.3 casos en promedio por AI), mientras que la menor presencia es propia de Ciencias Médicas (1.08 casos). Nuevamente, los extremos de frecuencia de uso de las categorías de autocita están marcados por una ciencia humana y una ciencia pura.

En la Figura 2 presentamos los resultados según la experticia de los autores. Como se observa, el uso de la autocita es transversal a autores noveles y consolidados. Sin embargo, estos últimos utilizan con mayor frecuencia dicho mecanismo (4.28 veces por AI, en promedio). Estos datos no resultan extraordinarios, pues son los autores consolidados quienes colocan publicaciones 
previas para referenciar. Por el contrario, es poco esperable que en los AI producidos por noveles también haya una presencia importante de autocitas (1.61 casos promedio por AI), ya que no disponen de artículos previamente publicados en revistas indexadas. No obstante, como evidencian los datos, igualmente recurren a la práctica de referenciar sus trabajos previos, aun cuando ellos corresponden a publicaciones que se pueden clasificar como literatura gris: tesis, ponencias, etc.

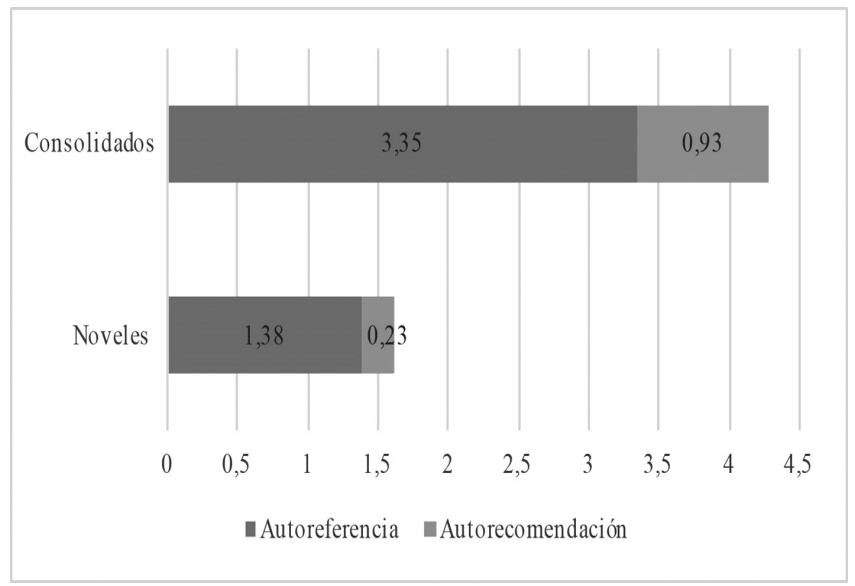

Figura 2. Promedio de autocitas según la experticia del autor

Probablemente, el uso de la autocita por los noveles se debe a que han aprendido que en su comunidad la autocita es una práctica válida y habitual, por lo que tratan de imitarla; y, por otro lado, porque les permitiría alcanzar el reconocimiento y validación de su comunidad meta. No obstante, dado que aún no poseen publicaciones en revistas indexadas, recurren a los trabajos con los que cuentan para mencionarlos cuantas veces sea posible en su primer AI indexado. Ejemplo de ello es el caso de una tesis de magíster que se referencia nueve veces en un artículo de 26 páginas [Hum_F_8] y el de la autocitación constante de una tesis de pregrado [CS_F_2071].

Esta dinámica, en la que los noveles reproducen las prácticas de los consolidados, la asociamos con la "teoría de las ventanas rotas" (broken windows theory) de Wilson y Kelling (1982). Según esta teoría, cuando las personas observan que otros quebrantan cierta norma o regla, es más probable que infrinjan otras, lo que hace que las transgresiones se propaguen. Análogamente, el uso frecuente de autocitas por parte de los expertos de la comunidad invitaría a los noveles a imitar esta práctica. 
En la Figura 3 presentamos la frecuencia de los tipos de autocita, considerando las dos variables integradas (área de la ciencia y experticia), lo que nos permitirá caracterizar la autocita más detalladamente.

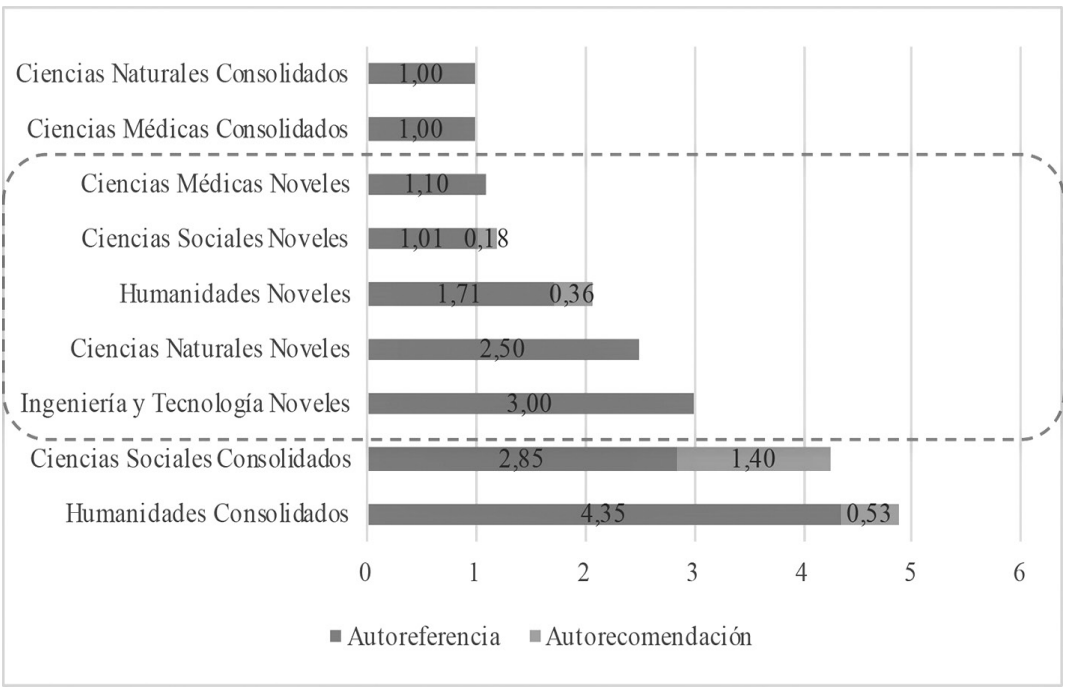

Figura 3. Promedio de autocitas por área de la ciencia y experticia de los autores

Al relacionar las dos variables estudiadas, si bien todos los investigadores utilizan estrategias de autocitación, es posible identificar un continnum entre los mecanismos utilizados por los autores según su experticia y disciplina. Así, en un extremo, se ubican los consolidados de Humanidades, quienes lideran en la frecuencia de uso de autocitas en general (4.88 casos por AI, en promedio) y también en la de autoreferencia (4.35); aunque no ocurre lo mismo con la autorecomendación, cuyo uso más frecuente lo ostentan los consolidados de Ciencias Sociales. En el otro extremo del continuum se ubican los consolidados de Ciencias Naturales y Ciencias Médicas, quienes despliegan, en promedio, una autocita por artículo. Este caso corresponde a la autoreferencia únicamente, pues sus textos prescinden de la autorecomendación.

Otro aspecto interesante de la Figura 3 (delimitado con una línea punteada) es el hecho de que, según el promedio de autocitas, todos los autores noveles, independientemente de su área de pertenencia, se ubican en el centro del continuum. Contrariamente, todos los consolidados se dispersan hacia la periferia, ya sea en el extremo de mayor o en el de menor uso de autocitas. A nuestro juicio, el hecho de que los autores consolidados sean los más propensos a autocitarse en las soft sciences podría explicarse a partir del "Efecto Mateo" (Merton, 1968) debido a que, en términos de Bunge (2002), este efecto 
encuentra su sustento en dos mecanismos: uno memorístico, pues se retiene mejor en la memoria el nombre del autor más conocido que el del no conocido, y otro de selección de los autores, pues se deposita más confianza en un autor por su currículum, pertenencia y validez en cierta comunidad.

\section{Conclusiones}

A partir de los resultados obtenidos, es posible afirmar que el autoreconocimiento que hace el autor de sus publicaciones es realizado mediante la autoreferencia y la autorecomendación, dos categorías con funciones distintas que permiten al autor posicionarse en la comunidad discursiva en la que se adscribe. El análisis de dichas categorías evidencia que la autocitación es un mecanismo presente en los AI, independientemente de la disciplina y del grado de experticia del autor. Así, mientras los consolidados la utilizan como mecanismo para mantener una línea temática de sus investigaciones o aumentar su visibilidad, los noveles copian esta práctica como validada y habitual de su comunidad académica.

A partir de nuestros hallazgos, podemos indicar, además, ciertas variaciones en su uso. Según el área de la ciencia, los investigadores de Humanidades son los que más frecuentan esta práctica discursiva. De acuerdo con el grado de experticia, los autores consolidados son quienes más recurren a la autoreferencia y a la autopromoción como estrategia de autocitación. Asimismo, y tomando en cuenta la integración de ambas variables, podemos afirmar que son los autores consolidados de Humanidades quienes más recurren a esta estrategia. Así, de acuerdo con Seeber et al. (2017), serían estos tipos de investigadores quienes realizan un uso oportunista de las autocitas para tener mayores probabilidades de éxito académico (asignación de fondos, adjudicación de proyectos concursables, contrataciones, etc.). Esta práctica, a pesar de ser cuestionable, podría traer grandes ventajas tanto para el investigador como para la revista en la que publica su trabajo (Anderson, Seashore y Earle, 1994).

En cuanto a las implicaciones de este estudio, consideramos que los resultados podrían ayudar a comprender el comportamiento de los científicos de distintas áreas de la ciencia y con distintos grados de experticia en el ámbito de las publicaciones científicas con las características de nuestra muestra. De igual manera, esta investigación permite identificar hasta qué punto la estrategia de la autocitación permite continuar la línea temática de un investigador (Campanario y Candelario, 2010; Gálvez, 2017; Reiter, Mimouni y Mimouni, 2015) o, simplemente, se emplea por vanidad del autor (Hyland y Jiang, 2018). Desde nuestro punto de vista, en cualquier caso, las autocitas 
son estrategias de posicionamiento del autor, ya sea para mostrar sus trabajos previos, evidenciar su línea investigativa, aumentar sus índices como investigador, lograr visibilidad o simple vanidad. Esto es más evidente en los casos de autorecomendación, en que el trabajo que se invita a revisar no siempre tiene relación directa con el tema tratado en el artículo donde presenta dicha recomendación.

De igual manera, los resultados presentados corroboran la idea que la autocita es una estrategia que permite conocer el comportamiento de los autores (Hyland, 2003). En nuestro caso, hemos evidenciado este comportamiento según el área de la ciencia y la experticia de los autores que utilizan dicho mecanismo.

A modo de proyección, nos parece interesante considerar, para investigaciones futuras, otras variables que nos permitan profundizar en el estudio de la autocita. Por ejemplo, el uso de autocitas en revistas de acceso libre y en aquellas que no lo son, o la variación de dicho recurso en revistas gold open access y green open access. Otro aspecto interesante sería determinar si la colaboración entre autores consolidados y noveles incrementa o no el grado de autocitación en las dos modalidades analizadas. Por último, sería de gran relevancia incluir la variable género (masculino o femenino) en nuestra aproximación al estudio de las autocitas.

Agradecimientos

Esta investigación fue realizada con el apoyo financiero del Proyecto FONDECYT de Postdoctorado N³150056 y del Programa PIA de la Dirección de Investigación de la Universidad de La Serena.

\section{REFERENCIAS}

Anderson, Melissa, Karen Seashore y Jason Earle. 1994. "Disciplinary and Departmental Effects on Observations of Faculty and Graduate Student Misconduct". The Journal of Higher Education 65 (4):331-350. doi: 10.1080/00221546.1994.11778504 Blessing, Lucienne y Amaresh Chakrabarti. 2009. DRM, a Design Research Methodology. Dordrecht: Springer.

Bunge, Mario. 2002. "El efecto San Mateo". Polis, Revista de la Universidad Bolivariana 1 (2). doi: http://dx.doi.org/10.32735/S0718-6568/2002-N2-167

Campanario, Juan y Antonia Candelario. 2010. "La influencia de las autocitas en el aumento del factor de impacto en revistas de Ciencias Sociales". Revista Española de Documentación Cientifica 33 (2): 185-200. doi: 10.3989/redc.2010.2.722 
Carlisle, Katherine, Joseph Sterbis, Phuong Do y Leah McMann. 2017. "Author self-citation in the urology literature". The Journal of Urology 197 (4): e928. doi: 10.1016/j.juro.2017.02.2302

Copez-Lonzoy, Anthony y Ángel Paz-Jesús. 2018. "Doppelgänger research? Considerations on self-citations". Revista de Psiquiatría y Salud Mental 11 (3): 185-186. doi: 10.1016/j.rpsmen.2018.01.001

Creswell, Jhon. 2007. "Qualitative Inquiry \& Research design. Choosing among five approaches”. 2a Ed. Thousand Oaks: Sage Publications.

Deschacht, Nick y Birgitt Maes. 2017. "Cross-cultural differences in self-promotion: A study of self-citations in management journals". Journal of Occupational and Organizational Psychology 90 (1): 77-94. doi: 10.1111/joop.12162

Fleiss, Joseph. 1971. "Measuring nominal scale agreement among many raters". Psychological Bulletin 76 (5):378-382. doi: 10.1037/h0031619

Fowler, James y Dag Aksnes. 2007. “Does self-citation pay?” Scientometrics 72 (3): 427-437. doi: 10.1007/s11192-007-1777-2

Freelon, Deen. 2010. "ReCal: Intercoder reliability calculation as a web service". International Journal of Internet Science 5 (1): 20-33.

Gálvez, Ramiro. 2017. "Assessing author self-citation as a mechanism of relevant knowledge diffusion”. Scientometrics 111 (3): 1801-1812. doi: 10.1007/s11192-017-2330-1

Ganga, Francisco, Lorena Paredes y Liliana Pedraja-Rejas. 2015. "Importancia de las publicaciones académicas: algunos problemas y recomendaciones a tener en cuenta”. Idesia 33 (4): 111-119. doi: 10.4067/s0718-34292015000400014

George-Nascimento, Mario. 2010. "Una evaluación de los índices bibliométricos I e Is de Molina-Montenegro \& Gianoli aplicada a investigadores en ciencias ecológicas en Chile". Revista Chilena de Historia Natural 83 (2): 229-235. doi: 10.4067/ S0716-078X2010000200003

Gul, Sumeer, Tariq Ahmad Shah y Huma Shafiq. 2017. "The prevalence of synchronous self-citation practices at the institutional level”. Malaysian Journal of Library EInformation Science 22 (1): 1-14. doi: 10.22452/mjlis.vol22no1.1

Henao, Carlos, Andrés Calvo y Hugo Gallego. 2017. "Análisis de Citas y Autocitas". Scientia et Technica 22 (2): 1- 4. doi: 10.22517/23447214.16541

Hernández, Roberto, Carlos Fernández y Pilar Baptista. 2006. Metodología de la investigación. 4a. ed. México: McGraw-Hill.

Hyland, Ken y Kevin Jiang. 2018. "Changing patterns of self-citation: cumulative inquirí or self-promotion?”. Text E Talk 38 (3):365-387. doi: 10.1515/text-2018-0004

Hyland, Ken. 2003. "Self-citation and self-reference: Credibility and promotion in academic publication". Journal of the American Society for Information Science and Technology 54 (3): 251-259. doi: 10.1002/asi.10204.7

Landis, Richards y Gary Koch. 1977. "The measurement of observer agreement for categorical data”. Biometrics 33 (1): 159-174. doi: 10.2307 / 2529310

Martínez, José y Laura Martínez. 2008. "Determinación de la máxima varianza para el cálculo del factor de imprecisión sobre la escala de medida, y extensión a diferentes tipos de muestreo". Psicothema 20 (2): 311-316.

Merton, Robert. 1968. “The Matthew Effect in Science”. Science 159 (3810): 56-63.

Meza, Paulina, Omar Sabaj y Ken Matsuda. 2017. "La autoría única en Scielo Chile: prácticas autoriales en las áreas de la ciencia”. Biblios: Journal of Librarianship and Information Science 66: 11-19. doi: 10.5195/biblios.2017.332 
Meza, Paulina. 2017a. Criterios de inclusión para la conformación del corpus CORAI ACEX. doi:10.13140/RG.2.2.10233.26720

Meza, Paulina. 2017b. "El Posicionamiento Estratégico del Autor en Artículos de Investigación: un modelo empíricamente fundado". Logos: Revista de Lingüística, Filosofía y Literatura 27 (1): 152-164. doi: 10.15443/rl2711

Meza, Paulina. 2018. "Estrategias de posicionamiento del Autor en Artículos de Investigación de Ciencias Sociales, Humanidades e Ingeniería: Novatos versus Expertos". Información tecnológica 29 (2): 3-18. doi: 10.4067/S07642018000200003

OCDE. 2007. Revised field of science and technology (FOS) classification in the Frascati Manual. https://www.oecd.org/science/inno/38235147.pdf

Reiter, Ofer, Michael Mimouni y Daniel Mimouni. 2015. "Analysis of self citation and impact factor in dermatology journals". International Journal of Dermatology 55 (9): 995-999. doi: 10.1111/ijd.13193

Sabaj, Omar y Dennis Páez. 2010. "Tipos y funciones de las citas en artículos de investigación de tres disciplinas”. Literatura y Lingüística 22: 117-134. doi: 10.4067/ S0716-58112010000200009

Salvador-Oliván, José, Gonzalo Marco-Cuenca y Rosario Arquero-Avilés. 2018. "Impacto de las revistas españolas de Biblioteconomía y Documentación y repercusión de las autocitas en su índice h”. Investigación Bibliotecológica 32 (77): 13-30. doi: 10.22201/iibi.24488321xe.2018.77.57852

Seeber, Marco, Mattia Cattaneo, Michelle Meolii y Paolo Malighettii. 2017. "Self-citations as strategic response to the use of metrics for career decisions". Research Policy 48 (2): 478-491. doi: 10.1016/j.respol.2017.12.004

Tognini-Bonelli, Elena. 2001. Corpus Linguistics at Work. Amsterdam: John Benjamins.

Wilson, James y George Kelling. 1982. "Broken windows”. Atlantic monthly 249 (3): 29-38.

Para citar este texto:

Meza, Paulina y Gustavo Ortega. 2019. "La autocita en artículos de investigación publicados en Scielo Chile: variación según el área de la ciencia y la experticia del autor". Investigación Bibliotecológica: archivonomía, bibliotecología e información 33 (81): 41-56.

http://dx.doi.org/10.22201/iibi.24488321xe.2019.81.58069 